\title{
Optimization and stability of a high-gain harmonic generation seeded oscillator amplifier
}

\author{
Georgia Paraskaki๑, Vanessa Grattoni, Tino Lang, and Johann Zemella \\ Deutsches Elektronen-Synchrotron DESY, 22607 Hamburg, Germany \\ Bart Faatz \\ Shanghai Advanced Research Institute, Chinese Academy of Sciences, Shanghai 201210, China \\ Wolfgang Hillert@ \\ University of Hamburg, 22761 Hamburg, Germany
}

(Received 14 November 2020; accepted 8 February 2021; published 3 March 2021)

\begin{abstract}
The free-electron laser (FEL) community is interested in taking full advantage of the high-repetitionrates of FELs run by superconducting machines while maintaining the spectral properties achieved with external seeding techniques. Since the feasibility of seed lasers operating at a repetition-rate of $\mathrm{MHz}$ and with sufficient energy in a useful wavelength range, such as the ultraviolet (UV) range is challenging, a seeded oscillator-amplifier scheme is proposed instead for generation of fully coherent and high-repetitionrate radiation. The process is triggered by an external seed laser while an optical feedback system feeds the radiation back to the entrance of the modulator where it overlaps with the next electron bunch. Downstream from the feedback system, the electron bunches are then used for harmonic generation. We discuss the optimization of dedicated simulations and we investigate the stability of this scheme with numerical simulations. As a result, we address the control of the reflectivity of the resonator as a key parameter to achieve a stable HGHG seeded radiation. Finally, we show the impact of the power fluctuations in the oscillator on the bunching amplitude with analytical and simulated results. The output FEL radiation wavelengths considered are $4.167 \mathrm{~nm}$ and $60 \mathrm{~nm}$.
\end{abstract}

DOI: $10.1103 /$ PhysRevAccelBeams.24.034801

\section{INTRODUCTION}

Cavity-based FELs are a well-established technology for generation of radiation in a wide range of wavelengths. Most of the FEL oscillators radiate in the terahertz (THz), infrared (IR), or in the visible wavelength range. Some examples are FELIX in the Netherlands [1], CLIO in France [2], FHI FEL in Germany [3], and FELiChEM in China [4]. There are other examples in different configurations, such as the inverse-Compton interaction compact $\mathrm{x}$-ray source in Hawaii [5] and the storage ring FEL at ELETTRA [6] which has achieved a wavelength down to $190 \mathrm{~nm}$. In addition, several simulation studies have explored low-gain FELs in the past decades $[7,8]$, however, the simulation codes have significantly improved since then, giving the possibility for more detailed studies.

\footnotetext{
"georgia.paraskaki@desy.de
}

Published by the American Physical Society under the terms of the Creative Commons Attribution 4.0 International license. Further distribution of this work must maintain attribution to the author(s) and the published article's title, journal citation, and DOI.
High-gain FEL Oscillators have been demonstrated in the past and are referred to as regenerative amplifier free electron lasers (RAFELs) [9,10]. Initially they addressed longer wavelengths with experimental tests in the IR [11], and later on they were proposed to be used in the x-ray regime as well [12]. A RAFEL requires only a few passes to reach saturation and the requirements on reflectivity are relaxed since it is a high-gain FEL and therefore, it consists of a low $\mathrm{Q}$ resonator. Another concept that is under investigation is the FEL oscillator (FELO) in the $\mathrm{x}$-ray wavelength range and down to $0.1 \mathrm{~nm}$ [13-15] as a direct source of radiation. There are different driver sources for such an XFELO. Originally, there were studies for an energy recovery linac and therefore, a low-gain FEL. Later, it was adapted to machines like the European XFEL [16] so that the gain could be larger. XFELOs aim at stable and fully coherent $\mathrm{x}$-ray radiation and operate with highly demanding Bragg crystals which require high reflectivity. At the same time there have been studies of schemes that use the oscillator as a source of seed instead of using the generated radiation directly [17-20], and suggestions on combining a RAFEL with harmonic generation as well [21]. 
In this paper, we describe a setup which uses a cavitybased FEL to imprint the energy modulation onto an electron beam for the implementation of a high-gain harmonic generation (HGHG) [22] seeding setup. The main ingredients of HGHG are a seed laser source, a modulator, a dispersive section, and a radiator. The seed laser interacts with the electron beam along a modulator with the result of modulating the energy of it sinusoidally with a periodicity matching the seed laser wavelength. Then, the dispersive section converts the energy modulation into a density modulation which has a frequency component at a certain harmonic of the seed laser. The prebunched electron beam is traversing the radiator which is tuned to be resonant at the same harmonic of the seed laser.

In single-pass seeded FELs, the seed source for the HGHG scheme is a laser which, when going to highrepetition-rates, determines the repetition-rate of the output seeded FEL radiation. As an example, currently at FLASH $[23,24]$ the seeding experiments are done at a repetitionrate of $10 \mathrm{~Hz}$, in single-bunch operation and at FERMI with a repetition rate of $50 \mathrm{~Hz}$ [25]. In the seeded oscillatoramplifier, the optical properties of such a seed laser are retained in a feedback system while the repetition-rate is no longer limited by the seed laser and is determined by the cavity, so it can be increased easily to a $\mathrm{MHz}$ and beyond. Seed laser sources with a MHz repetition-rate are currently under development, but are challenging systems. The requirements for tunable sources with tens of $\mu \mathrm{J}$ of pulse energy, excellent stability, less than $1 \%$ rms energy fluctuations and wavelength stability below $1 \% \mathrm{rms}$ with respect to the spectral bandwidth, make these lasers being considered as beyond the state of the art. Therefore, this scheme is proposed as an alternative solution for generation of high-repetition-rate seeded FEL radiation.

This scheme offers two advantages: the first one is that it can generate seeded FEL radiation at high-repetition-rates beyond the capability of the current seed laser systems exploited to generate ultra short pulses in the UV wavelength range and below. In addition to increasing the repetition-rate of HGHG, this scheme offers the possibility to extend the output wavelength range at this repetition-rate and achieve shorter wavelengths with HGHG. This is due to the amplification of the seed laser input intensity in the modulator which allows the use of shorter wavelength seed lasers of lower intensity. In addition, there are different possibilities offered by this scheme, which are discussed in [26]. In this paper, we show results of an HGHG seeded oscillator-amplifier which generates high-repetition-rate seeded FEL radiation which was first introduced in [27,28]. The simulation results here are more detailed and in addition, the optimization of the simulations is discussed and the stability of this scheme is investigated.

This approach overcomes the limitation of requiring high-repetition-rate seed lasers. This is crucial for pulsed machines, but it is also vital for future continuous wave (CW) machines. Currently, the x-ray user facilities FLASH at DESY, and the European XFEL are the only operating pulsed FELs driven by superconducting linear accelerators, while LCLS-II [29] and SHINE [30] will be CW machines and are under construction. Here, we use FLASH as an example, which operates in a burst-mode. This means that every tenth of a second a $800 \mu \mathrm{s}$ rf pulse is accelerated, containing a bunch train of 800 bunches. The repetitionrate of FLASH of $1 \mathrm{MHz}$ would require a cavity of $300 \mathrm{~m}$ roundtrip length, while for the $4.5 \mathrm{MHz}$ of the European XFEL this would be reduced to $66 \mathrm{~m}$.

\section{WORKING PRINCIPLE AND SIMULATION IMPLEMENTATION}

\section{A. The HGHG seeded oscillator-amplifier scheme}

The goal with the cavity in this scheme is to increase the low-repetition-rate of the seed laser to seed the highrepetition-rate bunches of a superconducting FEL. The process is initiated by a low-repetition-rate seed laser for the first pass, in the case of a burst-mode. The power of the laser is amplified by its interaction with the next electron bunch in the in-cavity undulator, the modulator, and is then stored in an optical cavity in order to seed the next electron bunch arriving at the modulator.

The energy-modulated electron beam exits the modulator and traverses a chicane with a longitudinal dispersion $\left(R_{56}\right)$ which induces a density modulation and hence, microbunches with high harmonic content are formed. Downstream of the chicane, an undulator called an amplifier is placed. The amplifier is resonant with the desired harmonic of the seed laser which corresponds to the wavelength of the output seeded FEL radiation. Therefore, this scheme is comparable to a regular single-pass HGHG scheme [31]. The only difference is that it requires a longer modulator in order to amplify the radiation in cavity field to compensate for the power losses that occur in the cavity. A schematic layout of the described HGHG seeded oscillator-amplifier set-up is shown in Fig. 1.

\section{B. Simulation setup and parameter choice}

Numerical simulations are implemented by combining two codes for the two different processes: the FEL process in the modulator with Genesis [32] with three-dimensional

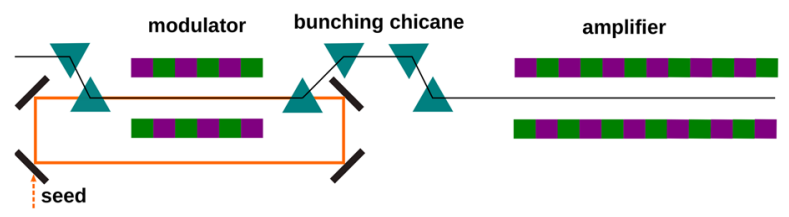

FIG. 1. Simplified schematic view of an HGHG seeded oscillator-amplifier. The orange line represents the path of the light pulse in the cavity and the black one the electron beam trajectory. 
time-dependent simulations, and the light propagation in the cavity with ocelot [33]. Genesis simulates the FEL process in the modulator and then, the three-dimensional field at the end of the modulator is extracted and is loaded into ocelot. In our simulations, ocelot is propagating the field along a drift, focuses transversely, and propagates again back to the entrance of the modulator. The field amplitude is reduced to account for cavity losses which are different for the two wavelengths and the longitudinal position of the field is changed so that the roundtrip of the cavity matches the repetition rate of the electron bunches and thus, overlaps optimally with the electron bunches in the modulator. This procedure is repeated for an arbitrary number of passes. Similar three-dimensional and timedependent simulation approaches have been adopted in other RAFEL designs [21,34].

For the simulations, we use the two electron beam energies $(0.75 \mathrm{GeV}$ and $1.35 \mathrm{GeV})$ that are proposed for the upgrade of FLASH [35] within the FLASH2020+ project [36]. The simulations shown here have been performed for two seed laser wavelengths (300 $\mathrm{nm}$ and $50 \mathrm{~nm}$ ) and their 5th and 12th harmonics respectively. Hence, the output FEL wavelengths are $60 \mathrm{~nm}$ and $4.167 \mathrm{~nm}$. This is because these are the longest and shortest foreseen wavelengths in the current design of FLASH2020+ in a seeded operation [36]. For the $50 \mathrm{~nm}$ seed laser a high harmonic generation (HHG) source is assumed [37], with state-of-theart possibilities demonstrated in the extreme ultraviolet (XUV) range [38,39].

The main simulation parameters are summarized in Table I. A flat-top electron beam current distribution is assumed and the modulator parameters are chosen to provide maximum power gain in the steady state region, which refers to the passes in the oscillator in equilibrium state. For the first pass, an ideal Gaussian seed laser pulse is used, which is then propagated and amplified selfconsistently during subsequent passes. Since the modulator length is the same for both wavelengths, the input power and reflectivity needed for $50 \mathrm{~nm}(3 \mathrm{MW}$ and $6 \%$ respectively) are higher than those for $300 \mathrm{~nm}(0.75 \mathrm{MW}$ and $1.01 \%$ respectively) since for shorter wavelengths the power gain length is longer [40].

Here we have assumed a simple resonator design that allows us to study in detail the FEL process and does not bound us to a specific optics selection and cavity design that would otherwise be necessary and crucial for the actual implementation of this scheme in an accelerator. We assume that a ring resonator would be a suitable and valid option for the resonator, therefore the total reflectivity applied in each pass accounts for the losses of all 4 elements and the focusing element in ocelot allows us to adjust the properties of the radiation field that would otherwise naturally diverge. As seen in Table I, at $300 \mathrm{~nm}$ the total reflectivity is $1.01 \%$, therefore each mirror should have a reflectivity of roughly $30 \%$, while
TABLE I. Parameters used for simulations. The rms undulator parameter is referred to as $K_{\mathrm{rms}}$, the number of undulator periods as $N_{u}$, and the undulator period as $\lambda_{u}$.

\begin{tabular}{lcc}
\hline \hline & \multicolumn{2}{c}{ Electron beam } \\
\hline Energy & $750 \mathrm{MeV}$ & $1350 \mathrm{MeV}$ \\
Uncorrelated energy spread & $120 \mathrm{keV}$ & $120 \mathrm{keV}$ \\
Peak current & $1 \mathrm{kA}$ & $1 \mathrm{kA}$ \\
Charge & $100 \mathrm{pC}$ & $100 \mathrm{pC}$ \\
Normalized Emittance & $1 \mathrm{~mm} \mathrm{mrad}$ & $1 \mathrm{~mm} \mathrm{mrad}$ \\
\hline \hline
\end{tabular}

\begin{tabular}{lcc}
\hline \hline & \multicolumn{2}{c}{ Input seed laser } \\
\hline Wavelength & $300 \mathrm{~nm}$ & $50 \mathrm{~nm}$ \\
Peak power & $0.65 \mathrm{MW}$ & $3 \mathrm{MW}$ \\
Pulse energy & $53 \mathrm{~nJ}$ & $250 \mathrm{~nJ}$ \\
FWHM Duration & $78 \mathrm{fs}$ & $78 \mathrm{fs}$ \\
\hline \hline
\end{tabular}

\begin{tabular}{lcc}
\hline \hline & \multicolumn{3}{c}{ Modulator } \\
\hline$K_{\mathrm{rms}}$ & 4.517 & 3.248 \\
$N_{u}$ & 90 & 90 \\
$\lambda_{u}$ & $60 \mathrm{~mm}$ & $60 \mathrm{~mm}$ \\
\hline \hline
\end{tabular}

\begin{tabular}{lll}
\hline \hline & \multicolumn{2}{c}{ Cavity } \\
\hline Reflectivity & $1.01 \%$ & \\
\hline \hline
\end{tabular}

for the $50 \mathrm{~nm}$ the total reflectivity is $6 \%$ and each mirror should have roughly $50 \%$ reflectivity. It should be noted that the exact wavelengths and tunability range for this scheme can be adapted based on availability in seed laser sources and mirrors as the technology advances, therefore the numbers presented here serve as an example study. In addition, the modulator length can be increased to compensate for higher resonator losses in case it is required by mirror availability.

\section{SIMULATION CONSIDERATIONS}

In the following section, the most important design considerations during the optimization process are described. The setup of the seeding parameters, the modulator length, and the cavity detuning are presented in Sec. III A, III B, and III C, respectively. In this section, we show results only for a modulator set to resonance with a $50 \mathrm{~nm}$ seed laser. This case is presented in more detail because it is one of the most challenging ones compared to longer wavelengths, since the power gain length at this wavelength is the longest, while the cavity losses are the highest and the technology in seed lasers is more limited. For the $300 \mathrm{~nm}$ resonant modulator, the optimization steps are the same, while the requirements on laser and mirror technology are more relaxed.

\section{A. Optimization of seeding parameters}

Once a stable operation in the longitudinal and transverse plane has been achieved, one can fine-tune the parameters 
for seeding. The optimal optimization process takes into account the target harmonic, and based on this, the energy modulation at the exit of the modulator is determined. The amplitude $\Delta E$ of the energy modulation induced by a field of peak power $P_{\text {mod }}$ in the modulator is approximately calculated as [41]:

$$
\Delta E=\sqrt{\frac{P_{\mathrm{mod}}}{P_{o}}} \frac{m_{e} 2 K L_{u} \mathrm{JJ}}{\gamma w_{0}},
$$

where $w_{0}$ is the laser waist size, $K$ is the dimensionless undulator parameter, $L_{u}$ is the undulator length, $m_{e}$ is the electron mass and $P_{0} \approx 8.7 \mathrm{GW}$ is a constant calculated in [41]. We define JJ as the difference of the Bessel functions, thus $\mathrm{JJ}=J_{0}(\xi)-J_{1}(\xi)$, where $\xi=K^{2} /\left(4+2 K^{2}\right)$. It should be noted that the simulations are performed with longer modulators than commonly used in seeding schemes, as it will be discussed in Sec. III B. In this case the power level along the modulator cannot be considered constant anymore and diffraction and slippage effects become important. In our case, Eq. (1) is valid with less than $2 \%$ error with $P_{\text {mod }}$ being the power level established after two thirds of the modulator length $P_{\text {mod, } 2 / 3}$. Assuming that the length of the modulator is fixed and the longitudinal and transverse properties of the radiation pulse are stable, one can readjust the power of the radiation field of the input seed laser on its first pass in the modulator to achieve precisely the energy modulation desired in the steady state regime.

After this step, the precise mean energy modulation in the steady state regime is calculated, since at this point the operation of the oscillator is stabilized and fixed. A useful parameter is the normalized energy modulation amplitude which is defined as [41]:

$$
A=\frac{\Delta E}{\sigma_{E, i}},
$$

where $\sigma_{E, i}$ is the energy spread upstream the modulator and $\Delta E$ is calculated with Eq. (1). Based on the energy modulation we optimize the bunching by adjusting the $R_{56}$. More commonly, the normalized dispersion is used which is defined as [41]:

$$
B=\frac{2 \pi R_{56} \sigma_{E, i}}{\lambda_{\bmod } E},
$$

where $\lambda_{\text {mod }}$ is the central wavelength of the radiation pulse at the modulator and $E$ is the electron beam energy. The bunching factor, which quantifies the density modulation within the bunch for a specific harmonic number $n$ is defined as [22]:

$$
b_{n}=\left|J_{n}(-n A B)\right| \exp \left(-\frac{1}{2} n^{2} B^{2}\right),
$$

where $J_{n}$ is the Bessel function of the first kind. Based on this formula, the working point, which is defined by the $A$ and $B$ parameters, is determined. As an example, one can aim for $20 \%$ of bunching amplitude at the 5 th harmonic of a $300 \mathrm{~nm}$ seed laser by adjusting the dimensionless parameters $A$ and $B$.

\section{B. Requirements for modulator length}

In this section, we discuss the importance of the length of the modulator and the power level of the radiation field in the cavity. This is because the required normalized energy modulation $A$ can be achieved by varying these two knobs, as shown in Eq. (1).

The upper limit for the modulator length is mainly imposed by the maximum energy spread which can be induced along the modulator while a high-quality beam, suitable for exponential amplification is still maintained. The energy spread of the electron beam upstream from the amplifier, $\sigma_{E}$, as a fraction of the electron beam energy $E$ should be much smaller than the parameter $\rho$. Therefore, $\sigma_{E} / E \ll \rho$ [42], where $\rho$ is the dimensionless fundamental FEL parameter [43].

The lower limit is imposed by the energy modulation needed to achieve significant bunching at a certain harmonic of the seed laser, and in addition, by the losses of the resonator. For HGHG, the normalized energy modulation $A$ at the end of the modulator should be approximately equal to the harmonic number in order to efficiently suppress the exponential term and maximize the Bessel function in Eq. (4). However, for higher harmonics it is preferred to deviate from this rule and use smaller energy modulations due to the energy spread limitations discussed above.

The following study for achieving the 12th harmonic of a $50 \mathrm{~nm}$ seed source wavelength is based on the previously illustrated principles. Based on this case, the length of the modulator is determined. For longer wavelengths, this length is sufficient and for lower harmonics the peak input power can be decreased to control the energy modulation. The requirements are: (1) For the simulation parameters in Table I, the FEL parameter at the amplifier is $\rho=1.3 \times 10^{-3}$, therefore the requirement $\sigma_{E} / E \ll \rho$ leads to $\sigma_{E} \ll 1.75 \mathrm{MeV}$, which is equivalent to $A \ll 14.6$. We set the limit at: $\boldsymbol{A} \leq \mathbf{1 0}$ for the energy modulation at the end of the modulator. (2) The maximum possible peak power of a seed laser at $50 \mathrm{~nm}$ and at $10 \mathrm{~Hz}$ is assumed to be $60 \mathrm{MW}$ : $\boldsymbol{P}_{\text {seed }} \leq 60 \mathrm{MW}$ [37]. (3) The maximum total reflectivity of a resonator for $50 \mathrm{~nm}$ is assumed to be $10 \%: \boldsymbol{R} \leq \mathbf{1 0} \%$ [44]. (4) The minimum normalized energy modulation for achieving at least $2 \%$ bunching is calculated analytically with Eq. (4) as: $\boldsymbol{A} \geq \mathbf{5 . 5}$.

Figure 2 shows the possible modulator lengths for different input radiation pulse peak powers, based on simulation results of a single pass in the modulator. As discussed, a maximum input peak power of $60 \mathrm{MW}$ is used. The requirement for $5.5 \leq A \leq 10$, reduces the allowed 


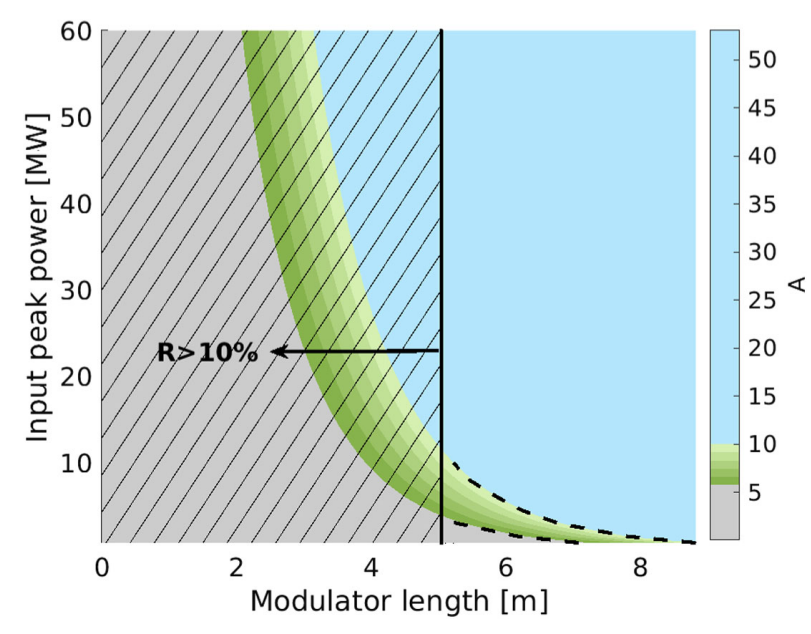

FIG. 2. Overview of possible modulator lengths and seed laser powers. The color bar shows the range of normalized energy modulation that is useful $(5.5 \leq A \leq 10)$ in shades of green. The grey and light blue areas of the color bar correspond to non acceptable values of energy modulation. The modulator length for which the power gain is not sufficient to cover resonator losses that are above $90 \%$ is also excluded and is shown in the plot with the diagonal black stripes. The small space of input peak power and modulator length combinations that is meeting all the requirements lies in between the two dashed black lines, and the vertical black line. As a result, the minimum possible modulator length is $5 \mathrm{~m}$.

parameter settings to the area indicated by the green band shown in the figure. In order to have enough energy modulation and at the same time achieve the same energy modulation in subsequent passes, the power has to be increased 10 times to compensate for the 90\% losses assumed. As a result, the limitation in reflectivity, $R \leq 10 \%$, reduces further the allowed parameter settings to the nonstriped area. In the end, there is only a small space available for combinations of seed laser power and modulator length that meet all four requirements, which is the area in between the black dashed lines and the vertical black line. It is concluded that in order to achieve the 12th harmonic of a $50 \mathrm{~nm}$ seed laser with reasonable bunching, realistic seed laser power and resonator total reflectivity, the modulator has to be at least $5 \mathrm{~m}$ long. Longer modulators can be used in case the reflectivity of $10 \%$ cannot be reached, without violating the other 3 conditions. This conclusion is valid for the specific parameters of the study and one can deviate for different simulation parameters, such as the electron beam parameters, or the focusing and waist size of the radiation pulse, for instance.

Based on this study, a modulator of $5.4 \mathrm{~m}$ length is chosen with an input seed laser peak power of $3 \mathrm{MW}$ and with total resonator reflectivity of $R=6 \%$ for the $50 \mathrm{~nm}$ case. The peak power in the steady state region is stabilized at 3.5 MW. We choose a longer than the minimum required modulator length of $5 \mathrm{~m}$ because this way, when there are deteriorations, such as a timing jitter between the seed laser, or a spatial jitter between them, there is still enough power gain to compensate for the resonator losses.

This modulator length is well beyond the traditional approach of using modulators of less than two gain lengths [22]. The consequence is that the modulator operates in the exponential regime, where the system is more sensitive to intensity fluctuations. The calculated power gain length for the simulations based on fitting the Ming-Xie formulas [43] is $1.12 \mathrm{~m}$ for the $50 \mathrm{~nm}$ resonant modulator and hence, the modulator covers roughly 4.8 gain lengths.

\section{Cavity detuning for optimum longitudinal overlap between electrons and stored light pulse}

Since the electrons are slower than the photon pulse within the modulator, due to their smaller longitudinal velocity, the laser pulse advances longitudinally in each pass, an effect known as slippage. When the group velocity of the electromagnetic wave is equal to the speed of light [45], the slippage is the product of the number of undulator periods $N_{u}$ and the resonant wavelength $\lambda_{\text {mod }}$, therefore $\Delta z=N_{u} \lambda_{\text {mod }}$. For high-gain FELs in the exponential gain regime, the group velocity is reduced and the slippage drops to $\Delta z=N_{u} \lambda_{\text {mod }} / 3$ as shown in $[34,45,46]$.

In this paper, the zero detuning $\Delta L_{\text {cav }}=0$ is defined as the length of the cavity for which the light pulse overlaps with the following electron bunch without taking into account the slippage. However, since there is slippage the synchronism of the system is achieved for a cavity length that is longer than the zero-detuning length, so $\Delta L_{\text {cav }}>0$. Figure 3 shows a detuning curve with the pulse energy and FWHM pulse duration averaged over 30 passes and a modulator resonant with $\lambda_{\text {mod }}=50 \mathrm{~nm}$. The cavity detuning can therefore be used to control the pulse duration, bandwidth and pulse energy of the output radiation [4]. In this paper, we are choosing a cavity length which maximizes the gain per pass and the pulse duration, which occurs when $\Delta L_{\text {cav }} \approx 54 \lambda_{\text {mod }}$. The width of the

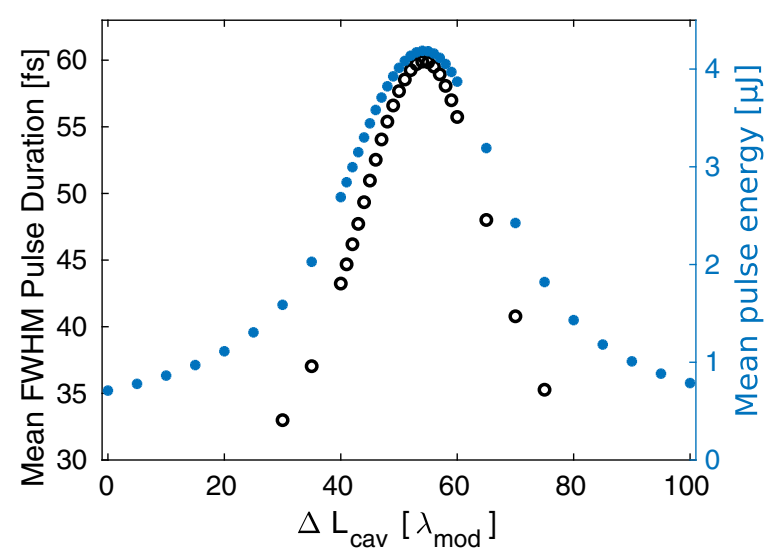

FIG. 3. Mean pulse energy and pulse duration over 30 passes in the oscillator for different cavity detuning $\Delta L_{\text {cav }}$ length. The modulator is tuned to $\lambda_{\text {mod }}=50 \mathrm{~nm}$. 
cavity detuning curve depends on the seed laser pulse duration and the electron bunch length which in this case is a 100 fs flat-top bunch.

\section{SIMULATION RESULTS}

\section{A. Transverse properties of light pulse in the cavity}

It results from the simulations that not only the longitudinal plane, but also the transverse plane of the radiation field plays an important role in the stability of the resonator, making the use of 3-dimensional codes such as Genesis of utmost importance. Changing the waist size and/or waist position within the modulator in one pass affects the gain of the system significantly. The Rayleigh length depends on the waist size, and its relation with the power gain length affects the energy exchange and amplification process in FELs when the diffraction effect cannot be suppressed [43]. In addition, the energy modulation depends on the effective power that overlaps temporally and spatially with the electron beam. In the transverse plane, the field has to be reasonably larger than the electron beam size, while it is usually desired to be shorter longitudinally.

The waist size in the modulator per pass is simulated and calculated with chi23d [47]. The $M^{2}$ [48] and waist size in the steady state region (here we assume from pass 30 to pass 100) for both horizontal and vertical planes along with their rms fluctuations and for resonators suitable for $50 \mathrm{~nm}$ and $300 \mathrm{~nm}$ are summarized in Table II. It should be noted, that the focusing has been optimized separately for the two wavelengths. The waist size $w_{0}$ is defined as the radius of the beam when the intensity drops to $1 / e^{2}$ of the on-axis intensity, and at focus position when propagating in a drift section. For the first pass, the seed laser is an ideal Gaussian pulse. The field stabilizes transversely in terms of intensity and waist size with very low fluctuations over the course of passages. We conclude that the radiation pulse has properties which depend on the design of the cavity. This has the advantage of compensating initial fluctuations and leading to a self-stabilized working point.

\section{B. Output seeded FEL radiation}

In this section we present the simulation results for 2 different cases: (i) The 5th harmonic of a modulator resonant with $300 \mathrm{~nm}$, (ii) and the 12th harmonic of a modulator resonant with $50 \mathrm{~nm}$. The stability per pass in

TABLE II. Transverse properties of stored radiation pulse: waist size and $M^{2}$ on horizontal (x) and vertical (y) plane and their rms fluctuations in the steady state region.

\begin{tabular}{lcc}
\hline \hline & $50 \mathrm{~nm}$ & $300 \mathrm{~nm}$ \\
\hline$M^{2} \mathrm{x}$ & $1.859 \pm 0.002$ & $1.538 \pm 0.002$ \\
$M^{2} \mathrm{y}$ & $1.576 \pm 0.002$ & $1.339 \pm 0.001$ \\
Waist size x & $(267.9 \pm 0.3) \mu \mathrm{m}$ & $(589.4 \pm 0.5) \mu \mathrm{m}$ \\
Waist size y & $(250.3 \pm 0.3) \mu \mathrm{m}$ & $(681.1 \pm 0.6) \mu \mathrm{m}$ \\
\hline \hline
\end{tabular}

frequency domain for the output FEL is shown in Fig. 4(a) and 5(a). As an example, the spectra of the passes 20, 40, 60, 80, 90, 100 for both cases are shown in Fig. 4(b) and 5 (b) and the power profiles for the same passes in Fig. 4(d) and 5(d) are shown separately. The evolution of the power profile for 100 passes is shown in Fig. 4(c) and 5(c).

For both output FEL wavelengths $(4.167 \mathrm{~nm}$ and $60 \mathrm{~nm}$ ), the spectrum is longitudinally coherent from the first pass already and shows wavelength and coherence stability, proving that one can use this scheme to generate fully coherent high-repetition rate seeded FEL radiation. For the $4.167 \mathrm{~nm}$ case, we see in Fig. 5(a) that there is a red shift in the spectrum which is less than $0.03 \%$, and depending on the experiment, can be tolerated. Finally, Fig. 5(c) shows a pulse shortening of the FEL pulse duration caused by the development of an FEL frequency chirp along the modulator with the number of passes. The pulse duration can be restored with a grating if required by the experiment. The timing for both cases seems to be reliable. Some of the basic parameters that characterize the final FEL pulses are shown in Table III as an average.

\section{STABILITY OF THE SYSTEM}

In this section, we investigate the stability of an HGHG seeded oscillator-amplifier. In Sec. VA we study the effect of fluctuations of the input seed laser power, the tolerances in the total reflectivity of the resonator, the effect of relative timing offset between the injected seed laser and the electron bunch in the first pass and the charge jitter. In all cases, the stability is examined with simulated results in terms of power gain $G$ and normalized energy modulation $A$ from the 30th pass and up to 100 passes in the oscillator, so in the steady state regime.

The power gain is defined as $G=\left(P_{f}-P_{i}\right) / P_{i}$, where $P_{i}$ is the peak power upstream from the modulator and $P_{f}$ is the peak power downstream of it. The results shown refer to a $50 \mathrm{~nm}$ resonant modulator and optical feedback system, since the $300 \mathrm{~nm}$ resonant modulator is operating at the exponential region as well and analogous results are observed in the simulations.

In Sec. V B, we discuss the effect of the oscillator power fluctuations on the energy modulation and the bunching amplitude, which is crucial for implementing the HGHG seeding. In this case, both the $300 \mathrm{~nm}$ and the $50 \mathrm{~nm}$ cases are considered and analytical derivations are compared to simulation results. Similarly to the previous section, we show the results for the 5 th harmonic of a $300 \mathrm{~nm}$ seed laser and the 12th harmonic of a $50 \mathrm{~nm}$ seed laser.

\section{A. Impact of input seed laser peak power, resonator reflectivity, laser-electron beam timing offset and charge jitter}

Shot-to-shot fluctuations of the input seed laser power at the first pass can affect the stability of the stored power in 


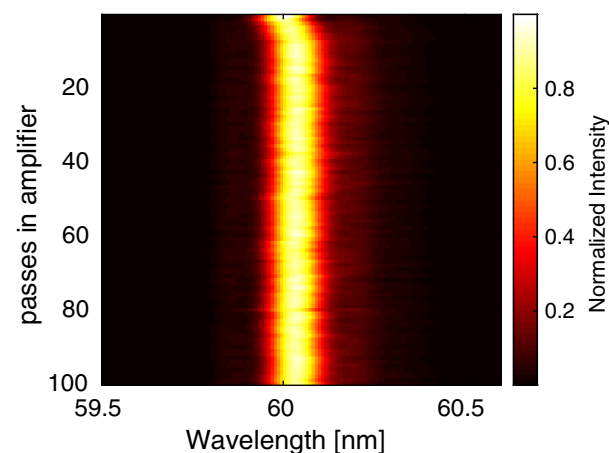

(a)

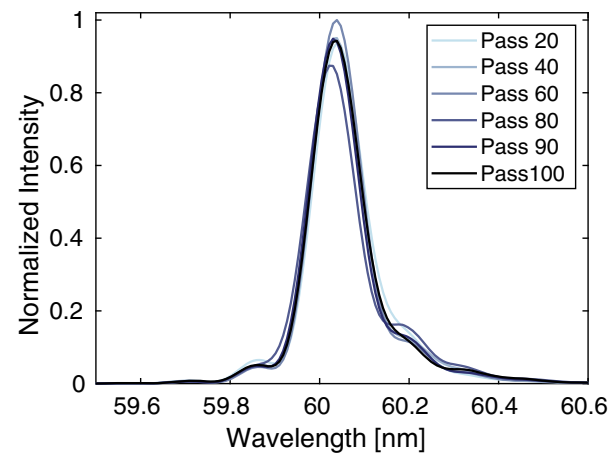

(b)

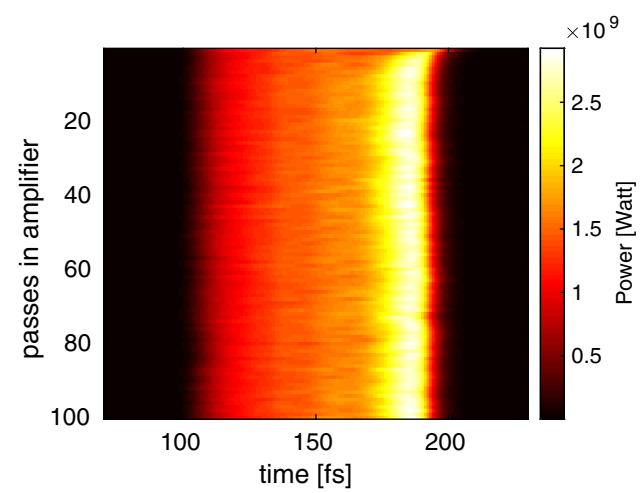

(c)

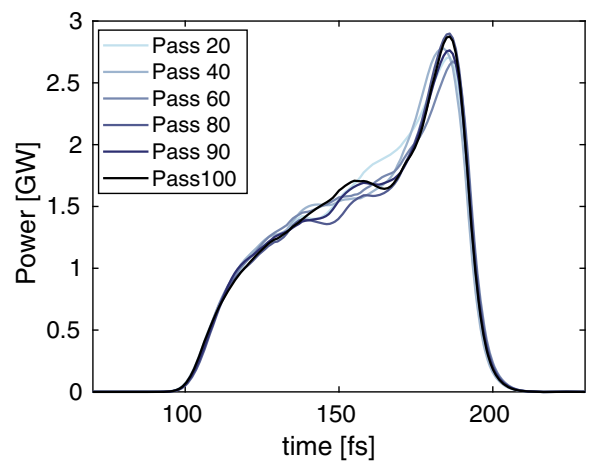

(d)

FIG. 4. 5th harmonic of a $300 \mathrm{~nm}$ seed light pulse. (a) Output spectrum per pass at amplifier. (b) Output spectrum for selected passes. (c) Power per pass in amplifier along the intrabeam coordinate $s$. (d) Output power profile for selected passes.

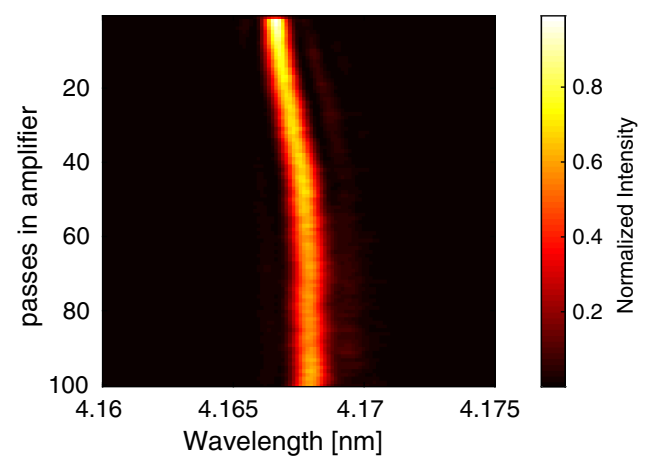

(a)

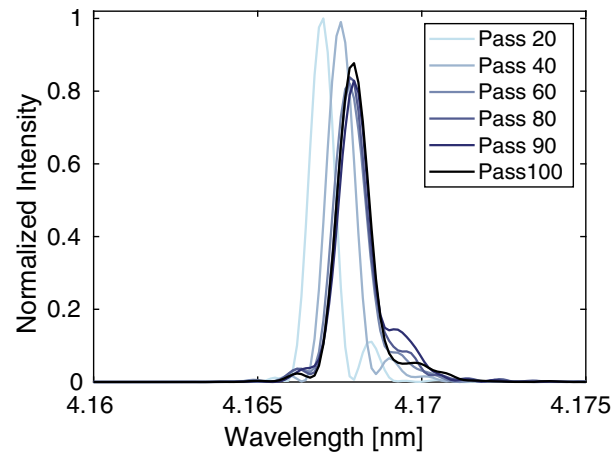

(b)

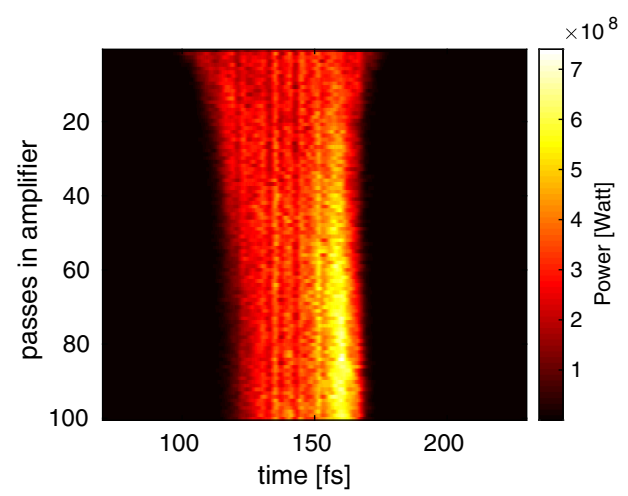

(c)

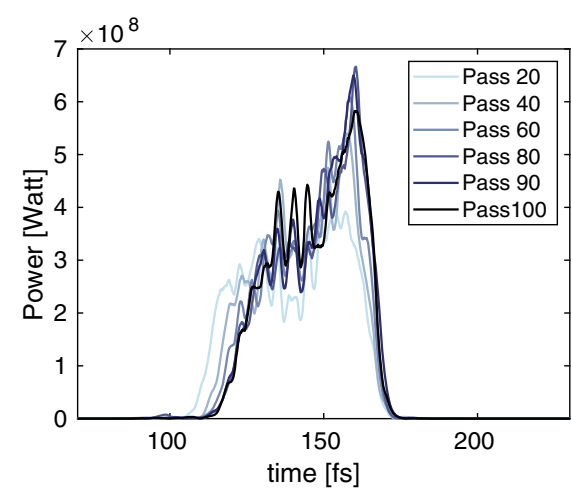

(d)

FIG. 5. 12th harmonic of a $50 \mathrm{~nm}$ seed light pulse. (a) Output spectrum per pass at amplifier. (b) Output spectrum for selected passes. (c) Power per pass in amplifier along the intrabeam coordinate $s$. (d) Output power profile for selected passes. 
TABLE III. Properties of FEL output radiation.

\begin{tabular}{lcc}
\hline \hline & $4.167 \mathrm{~nm}$ & $60 \mathrm{~nm}$ \\
\hline Pulse energy & $18.2 \mu \mathrm{J}$ & $150 \mu \mathrm{J}$ \\
rms pulse duration & $13.4 \mathrm{fs}$ & $25.2 \mathrm{fs}$ \\
$\Delta \lambda_{\text {rms }} / \lambda$ & $2.8 \times 10^{-4}$ & $1.8 \times 10^{-3}$ \\
\hline \hline
\end{tabular}

the feedback system per pass. In Fig. 6(a) the effect of the input seed laser power is shown for a $5 \%$ and $10 \%$ offset, which is considerably larger than the power stability of current seed laser systems which are often expected to be within $1 \%$ rms. Since a deviation of $10 \%$ in the seed laser power affects the energy modulation by less than $4 \%$ and the power gain considerably less than $0.01 \%$, it is concluded that the seed laser power fluctuations are not critical for the stability of the HGHG seeded oscillator-amplifier. This result relaxes the requirements on the seed laser source.

The reflectivity of the optical feedback system can vary either due to static effects or due to dynamic effects. Some of the effects that can degrade the operation of the cavity are the thermal loading, the slow degradation of the mirrors or the thermal effects on the mirror holders which can cause a cavity misalignment. Higher reflectivity, and therefore more power, leads to more instability as shown in Fig. 6(b). Increasing the power level of the radiation in the feedback system for a fixed modulator length results in a different working point on the gain curve which is closer to the saturation. In the reflectivity study case, the effect on both the relative energy modulation and the relative power gain is much stronger than in the seed power fluctuation study [Fig. 6(a)], since in this case the reflectivity offset is acting in every single pass and the effect is amplified with the number of passes.

It results that the reflectivity has to be carefully controlled and thermal effects have to be studied to secure a stable operation, since a $2 \%$ relative deviation in reflectivity can affect the energy modulation to an extent that HGHG is no longer possible. However, a careful design of the mirrors, and a feedback system that counteracts the degradation of the mirrors can ensure that the reflectivity changes are limited to acceptable ranges, which in our case should be $<1 \%$, and achieve active control.

Figure 6(c) shows the effect of a \pm 40 fs timing offset between the electron bunch and the seed laser which is injected in the first pass. The timing jitter should be better than 40 fs with optically locked seed lasers and assuming that feedback systems can prevent slow drifts. The simulations are implemented with a 100 fs flat-top electron bunch, since this bunch length is sufficient to simulate the overlap between the electron bunch and the radiation pulse, taking into account the slippage with accurate simulation results. This allows reducing the demanding required computational time needed for such simulations that run in several passes. However, only for this study, we have extended the electron bunch length to a $300 \mathrm{fs}$ flat-top current distribution, for realistic results of time jitter. In all cases, the energy modulation is not affected more than $4 \%$, therefore the timing between the electron bunch and the seed laser injected in the first pass is not crucial when the electron bunch is sufficiently long and uniform.

In addition, we simulated the effect of charge jitter up to $\pm 1 \%$ [49] by adjusting the peak current of the flat-top. The simulation results showed that even with the maximum offset, the energy modulation is affected by less than $4 \%$ and the gain by less than $0.2 \%$ within the error bars. It is concluded that a moderate charge jitter is not critical for the implementation of the scheme under study. In the following section, we extend the study to understand how the normalized energy modulation fluctuations affect the formation of the bunching amplitude and eventually, the seeding process.

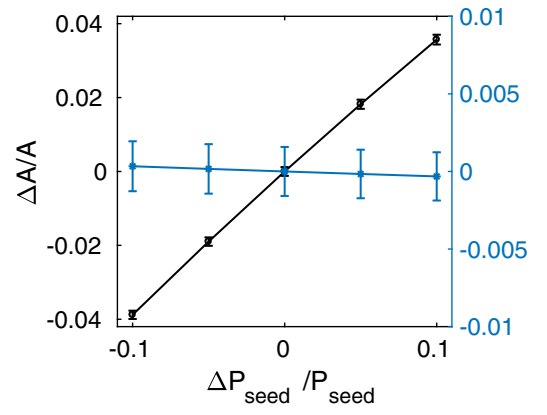

(a)

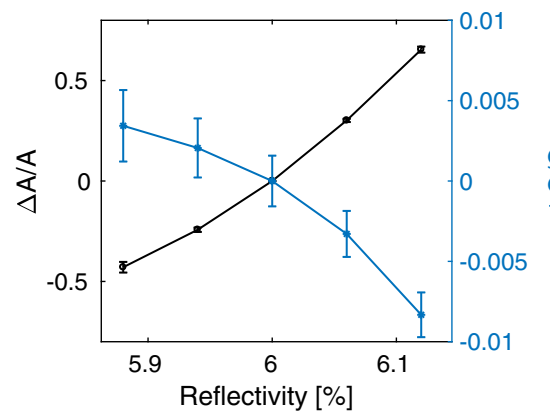

(b)

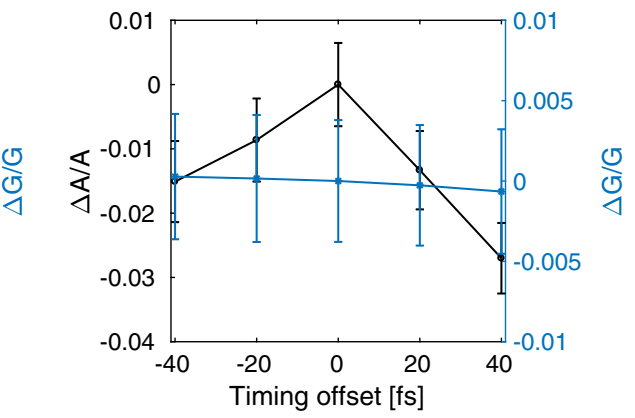

(c)

FIG. 6. Overview of the stability study. In (a) the effect of seed laser peak power, $P_{\text {seed }}$, fluctuation up to $\pm 10 \%$ is shown, in (b) the reflectivity tolerances for up to $\pm 2 \%$ and (c) the effect of timing offset between the external seed laser pulse and the electron beam for up to $\pm 40 \mathrm{fs}$. In all cases we show the effect on the relative normalized energy modulation $\Delta A / A$, achieved at the steady state in the oscillator (passes between 30 and 100), and on the right axis, the effect on the relative power gain, $\Delta G / G$ as an average over the same passes. The error bars indicate the standard error on the mean values calculated at the steady state region. 


\section{B. Sensitivity of bunching amplitude on power fluctuations}

As discussed in the previous section, there are several factors that can cause fluctuations in the power level, and hence the energy modulation achieved at the modulator. After exiting the chicane, the prebunched electron bunches arrive at high-repetition-rates and enter the amplifier. At this second stage, the amplifier, the only input parameter is the prebunched electron bunch. For this reason, it is essential to study the sensitivity of the bunching factor on the power fluctuations in the oscillator. Starting from Eq. (1), and Eq. (2), the sensitivity of the normalized energy modulation $A$ to the power per pass $P_{\bmod }$ of the stored radiation pulse in the cavity is

$$
\frac{\Delta A}{A}=\frac{1}{2} \frac{\Delta P_{\mathrm{mod}}}{P_{\mathrm{mod}}} .
$$

The rms power fluctuations and rms normalized energy modulation fluctuations are shown in Table IV and they are consistent with Eq. (5). In addition, Table V summarizes the seed laser pulse properties at the exit of the modulator, where the peak power maximizes. The peak power that reaches the mirror downstream will be less than the several MW reported here due to diffraction.

Based on Eq. (1), (2), and (4), the power fluctuations in the oscillator translate into bunching fluctuations for the electron beam that enters the amplifier. In turn, the power level $P_{\text {th }}$ when the power growth is transitioning to exponential in the amplifier is correlated with the bunching amplitude as: $P_{\text {th }} \propto\left|b_{n}\right|^{2}$ [50]. This means that for a fixed radiator length and sufficient initial bunching at the

TABLE IV. The Table summarizes the mean normalized energy modulation $A$ and the mean power in the cavity $P_{\text {mod }}$ over 100 passes with their rms fluctuations. In addition, the normalized dispersive strength $B$ used for the analytical calculations in this section is shown.

\begin{tabular}{lcc}
\hline \hline & $\begin{array}{c}300 \mathrm{~nm}-5 \text { th } \\
\text { harmonic }\end{array}$ & $\begin{array}{c}50 \mathrm{~nm}-12 \mathrm{th} \\
\text { harmonic }\end{array}$ \\
\hline$A$ & 7.24 & 7.11 \\
$A$ rms fluctuations & $1.66 \%$ & $1.93 \%$ \\
$P_{\text {mod }}$ rms fluctuations & $3.12 \%$ & $4.31 \%$ \\
$B$ & 0.17 & 0.14 \\
\hline \hline
\end{tabular}

TABLE V. Summary of laser pulse properties at the exit of the modulator.

\begin{tabular}{lcc}
\hline \hline & $300 \mathrm{~nm}-5$ th harm. & $50 \mathrm{~nm}-12$ th harm. \\
\hline$P_{\text {mod }}$ & $51.4 \mathrm{MW}$ & $56 \mathrm{MW}$ \\
Pulse energy & $3.4 \mu \mathrm{J}$ & $2.7 \mu \mathrm{J}$ \\
rms pulse duration & $25 \mathrm{fs}$ & $17.8 \mathrm{fs}$ \\
Beam radius x & $228 \mu \mathrm{m}$ & $147 \mu \mathrm{m}$ \\
Beam radius y & $192 \mu \mathrm{m}$ & $120 \mu \mathrm{m}$ \\
\hline \hline
\end{tabular}

amplifier, the bunching fluctuations can affect the final FEL power depending on whether or not saturation has been reached at this point.

We calculate the sensitivity of the bunching amplitude on energy modulation deviation for an HGHG scheme [51] as:

$$
\frac{\Delta b_{n}}{b_{n}}=n^{2} B^{2} \frac{\Delta A}{A}-\frac{1}{2}\left[n^{2}\left(B^{2}+B^{2} A^{2}-1\right)\right] \frac{\Delta A^{2}}{A^{2}} .
$$

One can easily extract the sensitivity of the bunching amplitude on the power fluctuations in the oscillator from Eq. (5) and (6).

Figure 7 shows how Eq. (6) relates to the simulation data for all 100 passes shown in Fig. 4 and 5. Table IV shows the exact parameters used in Eq. (6), with the normalized dispersion $B$ being calculated by taking into account the chicane strength and the longitudinal dispersion added along the long modulator [52]. The power fluctuations in the oscillator affect the bunching amplitude of higher harmonics more severely, based on Eq. (6) and the simulation results. We have extended the analytical study for up to a $40 \%$ deviation in $A$, in order to cover the effects studied in Sec. VA. These analytical calculations can be used to minimize the effect of the fluctuations in the oscillator on the bunching amplitude.

Finally, the bunching maps of the 5th harmonic of a $300 \mathrm{~nm}$ and the 12th harmonic of $50 \mathrm{~nm}$ seed laser are shown in Fig. 8(a) and 8(b). The peak to peak energy modulation fluctuations which occur in the first 100 passes are shown with a double arrow on the bunching maps The color bar indicates the analytically calculated bunching amplitude fluctuations expected for the calculated energy modulation fluctuations observed in the simulations and for

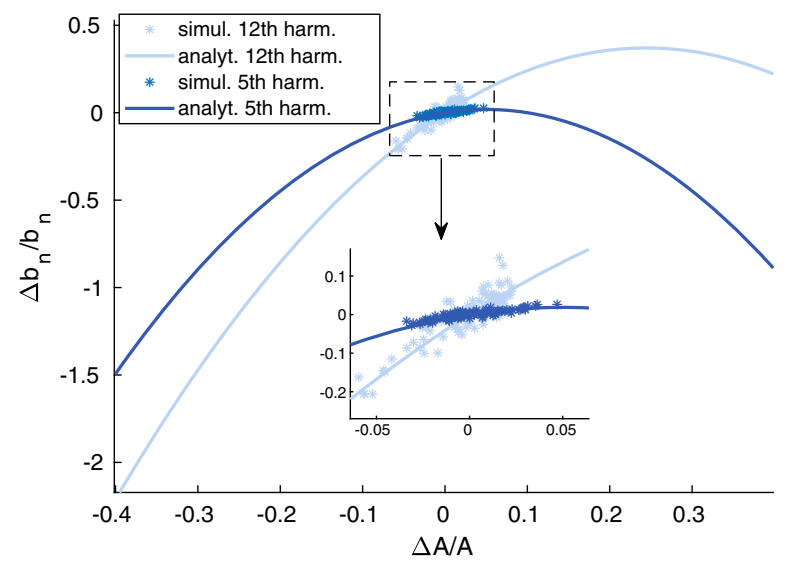

FIG. 7. Sensitivity of bunching amplitude on energy modulation fluctuations downstream from the modulator. The solid lines are calculated with Eq. (6), while the stars show the simulation results for 100 passes. The dark blue and the light blue color represent the 5th harmonic of a $300 \mathrm{~nm}$ resonant modulator and the 12th harmonic of a $50 \mathrm{~nm}$ resonant modulator, respectively. In the inset, we show the range of the fluctuations observed in the simulations in more detail. 


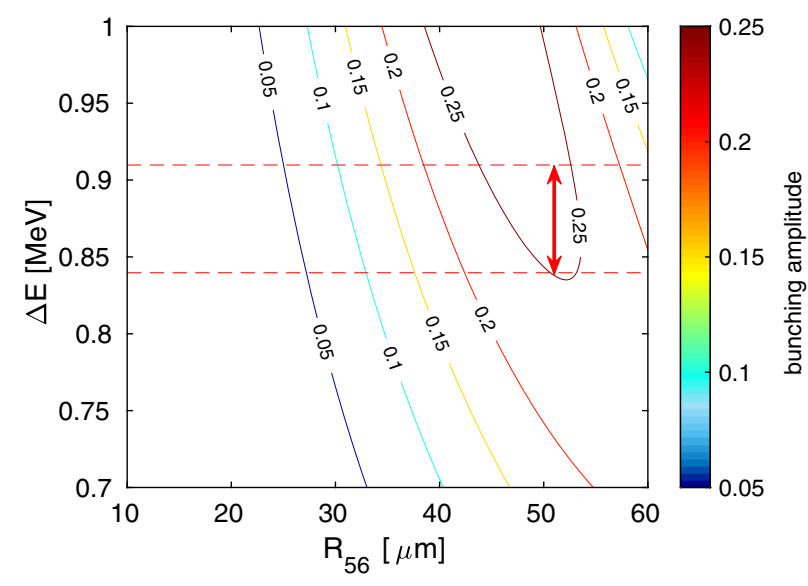

(a)

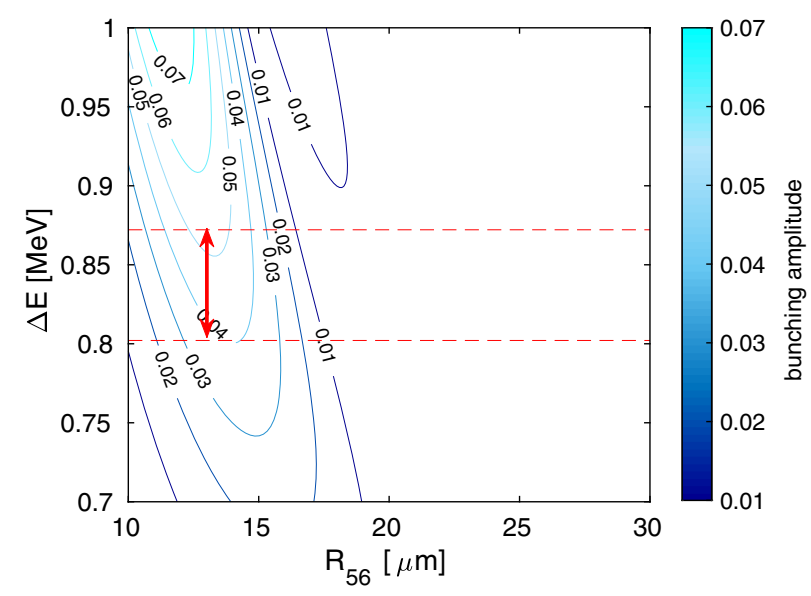

(b)

FIG. 8. Analytically calculated bunching maps [see Eq. (4)]. The red horizontal dashed lines show the peak to peak fluctuations calculated for 100 passes in the oscillator and the red double arrow indicates the bunching fluctuations for the $R_{56}$ used in the simulations. (a) 5 th harmonic of $300 \mathrm{~nm}$. (b) 12th harmonic of $50 \mathrm{~nm}$.

the $R_{56}$ set in the simulations. For increasing harmonic number the bunching maps become more constricted which illustrates the increasing sensitivity to fluctuations. One can use this type of plots to optimize for minimum bunching fluctuations, or for maximum bunching amplitude.

\section{CONCLUSIONS}

In this paper, we presented the optimization process of an HGHG seeded oscillator-amplifier. The modulator length was determined based on the requirements for the implementation of HGHG seeding and the technology available for this setting, and the seeding parameters were optimized. An overview of the output seeded FEL radiation per pass in time and frequency domain for 100 passes from initiation proves that fully coherent light can be generated almost immediately with a seeded oscillator-amplifier scheme.
Since the oscillator is partially operating in the exponential regime, the stability of the power per pass in the oscillator is more crucial compared to conventional FEL oscillators operating close to saturation. The seed laser power fluctuations at the first pass can be tolerated up to $10 \%$, which relaxes the requirements on the seed laser. In addition, a timing jitter between electron and laser beam up to $\pm 40 \mathrm{fs}$ and a charge jitter up to $\pm 1 \%$ can be tolerated, indicating the feasibility of the scheme. The reflectivity seems to be the main challenge since it should deviate considerably less than $1 \%$ from the nominal value for reliable results. This can be achieved with appropriate mirror choice and the use of feedback systems. An active control on the reflectivity is crucial, since it is the main tuning knob for compensation of other fluctuations and for system stability.

Finally, we show how the power fluctuations in the oscillator affect the fluctuations in energy modulation which in turn determine the fluctuations in bunching amplitude. Simulation results were compared with analytical expressions in good agreement and can be used to determine a stable working point.

\section{ACKNOWLEDGMENTS}

The authors would like to thank Sven Ackermann, Enrico Allaria, Francesca Curbis, Gianluca Geloni, Lucas Schaper, Takanori Tanikawa for their useful insights and Svitozar Serkez for his support with ocelot. We would like to thank Saša Bajt, Elke Plönjes, and Rolf Treush for information on mirrors. This work was supported by the Impuls- und Vernetzungsfond der Helmholtz-Gemeinschaft e.V. within the CAS-Helmholtz International Laboratory on Free-Electron Laser Science and Technology (CHILFEL), Grant No. InterLabs-0002 and by the Maxwell computational resources operated at Deutsches ElektronenSynchrotron (DESY), Hamburg, Germany.

[1] D. Oepts, A. van der Meer, and P. van Amersfoort, The free-electron-laser user facility FELIX, Infrared Physics \& Technology 36, 297 (1995).

[2] J. M. Ortega, The CLIO infrared FEL facility, Synchrotron Radiat. News 9, 20 (1996).

[3] W. Schöllkopf, S. Gewinner, H. Junkes, A. Paarmann, G. von Helden, H. P. Bluem, and A. M. M. Todd, The new IR and THz FEL facility at the Fritz Haber Institute in Berlin, in Advances in X-ray Free-Electron Lasers Instrumentation III, Vol. 9512, edited by S. G. Biedron, International Society for Optics and Photonics, Prague, Czech Republic (SPIE, 2015), pp. 238-250, https://spie.org/eoo/conferencedetails/ euv-X-ray-optics?utm_id=reoo21 scpw.

[4] Z.-Y. Zhao, H.-T. Li, and Q.-K. Jia, Effect of cavity length detuning on the output characteristics for the middle infrared FEL oscillator of FELiChEM, Chin. Phys. C 41, 108101 (2017).

[5] P. Niknejadi, J. M. Kowalczyk, M. R. Hadmack, B. T. Jacobson, I. Howe, S. Kan, S. Smith, E. B. Szarmes, 
G. Varner, and J. M. Madey, Free-electron laser inverseCompton interaction x-ray source, Phys. Rev. Accel. Beams 22, 040704 (2019).

[6] M. Trovò, J. Clarke, M. Couprie, G. Dattoli, D. Garzella, A. Gatto, L. Giannessi, S. Günster, N. Kaiser, M. Marsi, M. Poole, D. Ristau, and R. Walker, Operation of the European storage ring FEL at ELETTRA down to $190 \mathrm{~nm}$, Nucl. Instrum. Methods Phys. Res., Sect. A 483, 157 (2002).

[7] G. Dattoli, B. Faatz, L. Giannessi, and P. L. Ottaviani, The tandem FEL dynamic behavior, IEEE J. Quantum Electronics 31, 1584 (1995).

[8] G. Dattoli, L. Giannessi, and P. Ottaviani, Oscillatoramplifier free electron laser devices with stable output power, J. Appl. Phys. 95, 3211 (2004).

[9] B. Faatz, J. Feldhaus, J. Krzywinski, E. Saldin, E. Schneidmiller, and M. Yurkov, Regenerative FEL amplifier at the TESLA test facility at DESY, Nucl. Instrum. Methods Phys. Res., Sect. A 429, 424 (1999).

[10] H. P. Freund, P. J. M. van der Slot, and Y. Shvyd'ko, An $\mathrm{X}$-ray regenerative amplifier free-electron laser using diamond pinhole mirrors, New J. Phys. 21, 093028 (2019).

[11] D. C. Nguyen, R. L. Sheffield, C. M. Fortgang, J. C. Goldstein, J. M. Kinross-Wright, and N. A. Ebrahim, First lasing of the regenerative amplifier FEL, Nucl. Instrum. Methods Phys. Res., Sect. A 429, 125 (1999).

[12] Z. Huang and R. D. Ruth, Fully Coherent X-Ray Pulses from a Regenerative-Amplifier Free-Electron Laser, Phys. Rev. Lett. 96, 144801 (2006).

[13] K.-J. Kim, Y. Shvyd'ko, and S. Reiche, A Proposal for an X-Ray Free-Electron Laser Oscillator with an EnergyRecovery Linac, Phys. Rev. Lett. 100, 244802 (2008).

[14] J. Zemella, J. Rossbach, C. Maag, H. Sinn, and M. Tolkiehn, Numerical simulations of an XFELO for the european XFEL driven by a spent beam, in FEL 201234th International Free Electron Laser Conference, 429 (JACoW Publishing, Nara, Japan, 2012), https://accelconf .web.cern.ch/FEL2012/papers/wepd29.pdf.

[15] P. Rauer, I. Bahns, W. Decking, W. Hillert, J. Roßbach, and H. Sinn, Integration of an XFELO at the European XFEL facility, in Proc. FEL'19, Free Electron Laser Conference No. 39 (JACoW Publishing, Geneva, Switzerland, 2019), pp. 62-65, https://doi.org/10.18429/JACoW-FEL2019TUP009.

[16] D. Nölle, FEL operation at the European XFEL facility, in Proc. of International Free Electron Laser Conference (FEL'19), Hamburg, Germany, August 26-30, 2019, International Free Electron Laser Conference No. 39 (JACoW, Geneva, Switzerland, 2019).

[17] P. Gandhi, G. Penn, M. Reinsch, J. Wurtele, and W. Fawley, Oscillator seeding of a high gain harmonic generation free electron laser in a radiator-first configuration, Phys. Rev. Accel. Beams 16, 020703 (2013).

[18] V. Petrillo, A. Bacci, A. R. Rossi, L. Serafini, I. Drebot, M. R. Conti, M. Ruijter, M. Opromolla, S. Samsam, F. Broggi, G. Ghiringhelli, E. Puppin, G. Rossi, and A. tagliaferri, Coherent, high repetition rate tender X-ray Free-Electron Laser seeded by an Extreme Ultra-Violet Free-Electron Laser Oscillator, New J. Phys. 22, 073058 (2020).
[19] K. Li, J. Yan, C. Feng, M. Zhang, and H. Deng, High brightness fully coherent $\mathrm{X}$-ray amplifier seeded by a freeelectron laser oscillator, Phys. Rev. Accel. Beams 21, 040702 (2018).

[20] F. Ciocci, G. Dattoli, A. Angelis, F. Garosi, L. Giannessi, P. Ottaviani, and A. Torre, Design elements of a high-power VUV free electron laser, Il Nuovo Cimento Soc. Ital. Fis. 106A, 1757 (1993).

[21] B. W. J. McNeil, N. R. Thompson, D. J. Dunning, J. G. Karssenberg, P. J. M. van der Slot, and K.-J. Boller, A design for the generation of temporally-coherent radiation pulses in the VUV and beyond by a self-seeding highgain free electron laser amplifier, New J. Phys. 9, 239 (2007).

[22] L. H. Yu and J. Wu, Theory of high gain harmonic generation: an analytical estimate, Nucl. Instrum. Methods Phys. Res., Sect. A 483, 493 (2002).

[23] J. Rossbach, J. R. Schneider, and W. Wurth, 10 years of pioneering X-ray science at the Free-Electron Laser FLASH at DESY, Phys. Rep. 808, 1 (2019), 10 years of pioneering X-ray science at the Free-Electron Laser FLASH at DESY.

[24] B. Faatz, M. Braune, O. Hensler, K. Honkavaara, R. Kammering, M. Kuhlmann, E. Ploenjes, J. RoenschSchulenburg, E. Schneidmiller, S. Schreiber, K. Tiedtke, M. Tischer, R. Treusch, M. Vogt, W. Wurth, J. Zemella, and M. Yurkov, The FLASH facility: Advanced options for FLASH and future perspectives, Appl. Sci. 7, 1114 (2017).

[25] P. Ribič, A. Abrami, L. Badano, M. Bossi, H.-H. Braun, N. Bruchon, F. Capotondi, D. Castronovo, M. Cautero, P. Cinquegrana, M. Coreno, M. Couprie, I. Cudin, M. Danailov, D. N. Giovanni, A. Demidovich, S. Di Mitri, B. Diviacco, W. Fawley, and E. Allaria, Coherent soft $\mathrm{X}$-ray pulses from an echo-enabled harmonic generation free-electron laser, Nat. Photonics 13, 555 (2019).

[26] S. Ackermann, B. Faatz, V. Grattoni, M. M. Kazemi, T. Lang, C. Lechner, G. Paraskaki, J. Zemella, G. Geloni, S. Serkez, T. Tanikawa, and W. Hillert, Novel method for the generation of stable radiation from free-electron lasers at high repetition rates, Phys. Rev. Accel. Beams 23, 071302 (2020).

[27] G. Paraskaki, S. Ackermann, B. Faatz, V. Grattoni, C. Lechner, M. Mehrjoo, G. Geloni, S. Serkez, T. Tanikawa, and W. Hillert, Study of a seeded oscillator-amplifier FEL, in Proc. of International Free Electron Laser Conference (FEL'19), Hamburg, Germany, August 26-30, 2019, International Free Electron Laser Conference No. 39 (JACoW, Geneva, Switzerland, 2019).

[28] S. Ackermann, B. Faatz, V. Grattoni, C. Lechner, G. Paraskaki, G. Geloni, S. Serkez, T. Tanikawa, and W. Hillert, High-repetition-rate seeding schemes using a resonator-amplifier setup, in Proc. of International Free Electron Laser Conference (FEL'19), Hamburg, Germany, August 26-30, 2019, International Free Electron Laser Conference No. 39 (JACoW, Geneva, Switzerland, 2019).

[29] A. Brachmann, M. Dunham, and J. Schmerge, LCLS-II Status and upgrades, in Proc. FEL'19, Free Electron Laser Conference No. 39 (JACoW Publishing, Geneva, Switzerland, 2019), pp. 772-775, https://doi.org/10.18429/JACoW-FEL2019-FRA02. 
[30] T. Liu, X. Dong, and C. Feng, Start-to-end simulations of the reflection hard X-ray self-seeding at the SHINE project, in Proc. FEL'19, Free Electron Laser Conference No. 39 (JACoW Publishing, Geneva, Switzerland, 2019), pp. 254257, https://doi.org/10.18429/JACoW-FEL2019-TUP087.

[31] L.-H. Yu, M. Babzien, I. Ben-Zvi, L. F. DiMauro, A. Doyuran, W. Graves, E. Johnson, S. Krinsky, R. Malone, I. Pogorelsky, J. Skaritka, G. Rakowsky, L. Solomon, X. J. Wang, M. Woodle, V. Yakimenko, S. G. Biedron, J. N. Galayda, E. Gluskin, J. Jagger, V. Sajaev, and I. Vasserman, High-gain harmonic-generation free-electron laser, Science 289, 932 (2000).

[32] S. Reiche, GENESIS 1.3: a fully 3D time-dependent FEL simulation code, Nucl. Instrum. Methods Phys. Res., Sect. A 429, 243 (1999).

[33] I. Agapov, G. Geloni, S. Tomin, and I. Zagorodnov, OCELOT: A software framework for synchrotron light source and FEL studies, Nucl. Instrum. Methods Phys. Res., Sect. A 768, 151 (2014).

[34] H. P. Freund, D. C. Nguyen, P. A. Sprangle, and P. J. M. van der Slot, Three-dimensional, time-dependent simulation of a regenerative amplifier free-electron laser, Phys. Rev. Accel. Beams 16, 010707 (2013).

[35] J. Zemella and M. Vogt, Optics \& compression schemes for a possible FLASH upgrade, in Proc. 10th International Particle Accelerator Conference (IPAC'19), Melbourne, Australia, 19-24 May 2019, International Particle Accelerator Conference No. 10 (JACoW Publishing, Geneva, Switzerland, 2019), pp. 1744-1747, https://doi.org/ 10.18429/JACoW-IPAC2019-TUPRB026.

[36] M. Beye, FLASH2020+: Making FLASH brighter, faster and more flexible: Conceptual Design Report (Verlag Deutsches Elektronen-Synchrotron, Hamburg, 2020), pp. 1-126.

[37] E. Takahashi, Y. Nabekawa, and K. Midorikawa, Generation of $10-\mu \mathrm{J}$ coherent extreme-ultraviolet light by use of high-order harmonics, Opt. Lett. 27, 1920 (2002).

[38] C. Heyl, C. Arnold, A. Couairon, and A. L'Huillier, Introduction to macroscopic power scaling principles for high-order harmonic generation, J. Phys. B 50, 013001 (2017).

[39] I. Makos, I. Orfanos, A. Nayak, J. Peschel, B. Major, I. Liontos, E. Skantzakis, N. Papadakis, C. Kalpouzos, M. Dumergue, S. Kühn, K. Varju, P. Johnsson, A. L'Huillier, P. Tzallas, and D. Charalambidis, A 10-gigawatt attosecond source for non-linear XUV optics and XUVpump-XUV-probe studies, Sci. Rep. 10, 3759 (2020).

[40] P. Schmüser, M. Dohlus, and J. Rossbach, Ultraviolet and Soft X-Ray Free-Electron Lasers: Introduction to Physical Principles, Experimental Results, Technological
Challenges, 1st ed. (Springer Publishing Company, New York, 2008).

[41] E. Hemsing, G. Stupakov, D. Xiang, and A. Zholents, Beam by design: Laser manipulation of electrons in modern accelerators, Rev. Mod. Phys. 86, 897 (2014).

[42] S. Reiche, Overview of seeding methods for FELs, in Proceedings of the 4th International Particle Accelerator Conference, IPAC-2013, Shanghai, China, 2013 (JACoW, Shanghai, China, 2013), p. 2063.

[43] M. Xie, Design optimization for an X-ray free electron laser driven by SLAC LINAC, in Proceedings, 16th Particle Accelerator Conference and International Conference on High-Energy Accelerators, HEACC 1995: Dallas, USA, May 1-5, 1995, Conf. Proc. C950501, 183 (1996).

[44] M. Vidal-Dasilva, M. Fernández-Perea, J. A. Méndez, J. A. Aznárez, and J. I. Larruquert, Narrowband multilayer coatings for the extreme ultraviolet range of 50-92 nm, Opt. Express 17, 22773 (2009).

[45] H. Freund and T. Antonsen, Principles of Free-Electron Lasers (Springer, New York, 2018), pp. 328-330.

[46] X. Yang, N. Mirian, and L. Giannessi, Postsaturation dynamics and superluminal propagation of a superradiant spike in a free-electron laser amplifier, Phys. Rev. Accel. Beams 23, 010703 (2020).

[47] T. Lang, A. Harth, J. Matyschok, T. Binhammer, M. Schultze, and U. Morgner, Impact of temporal, spatial and cascaded effects on the pulse formation in ultrabroadband parametric amplifiers, Opt. Express 21, 949 (2013), available at www.chi23D.com.

[48] S. Ackermann, B. Faatz, and V. Miltchev, Modal analysis of a seeded free-electron laser, Phys. Rev. Accel. Beams 16, 100702 (2013).

[49] T. Kozak, B. Steffen, S. Pfeiffer, S. Schreiber, and A. Napieralski, Fast intra bunch train charge feedback fo $\rho$ FELs based on photo injector laser pulse modulation, IEEE Trans. Nucl. Sci. 64, 2904 (2017).

[50] L. Giannessi, Seeding and harmonic generation in freeelectron lasers (Synchrotron Light Sources and FreeElectron Lasers: Accelerator Physics, Instrumentation and Science Applications, 2016), pp. 195-223.

[51] E. Hemsing, B. Garcia, Z. Huang, T. Raubenheimer, and D. Xiang, Sensitivity of echo enabled harmonic generation to sinusoidal electron beam energy structure, Phys. Rev. Accel. Beams 20, 060702 (2017).

[52] R. Molo, Investigation of short-pulse radiation sources at DELTA based on coherent harmonic generation and echoenabled harmonic generation, Master's thesis, Technical University Dortmund, DELTA, 2011. 